\title{
Infección neonatal y tamizaje universal para Streptococcus agalactiae ( $\beta$-hemolítico del grupo B) a la mujer embarazada. Recomendación técnica
}

\author{
Comité Consultivo Infecciones Neonatales-CCIN \\ Sociedad Chilena de Infectología
}

\author{
Neonatal infection and universal screening for Streptococcus agalactiae \\ (group B $\beta$-hemolytic Streptococcus) in the pregnant woman. \\ Technical recommendation
}

S treptococcus agalactiae (comúnmente denominado Streptococcus $\beta$ - hemolítico del grupo B-SBHGB) emergió en los años setenta como la causa más común de sepsis y meningitis en niños bajo 3 meses de edad, con una tasa de mortalidad global que alcanzaba a $50 \%$, la que ha disminuido a $4-6 \%$ en los últimos años ${ }^{1}$. Actualmente sigue siendo una principal causa de infecciones graves en recién nacidos de término (RNT) y recién nacidos prematuros (RNPT), presentándose como sepsis, neumonía y/o meningitis, con una incidencia actual de $0,5 / 1.000$ recién nacidos vivos (RNV) en diversas latitudes $^{2,3}$, aunque existen diferencias geográficas en la epidemiología de la enfermedad (7-38/1.000 RNV en África y Asia a 0,5-3,5/1.000 RNV en Estados Unidos de América $)^{4,5}$.

En nuestro país no existe reporte de una tasa específica de incidencia y/o mortalidad de la enfermedad invasora neonatal, dado que la vigilancia no se realiza por notificación de casos clínicos, sino que por reporte de cepas provenientes de infecciones invasoras, desde los laboratorios locales al centro de referencia nacional: Instituto de Salud Pública.

Las infecciones invasoras por SBHGB se han clasificado como sepsis precoz (SP) si se presentan en los primeros siete días de vida o, sepsis tardía (ST) si ocurren después de la primera semana y hasta los tres meses de vida ${ }^{2}$.

La mortalidad de la SP se sitúa alrededor de $12 \%$, siendo superior a la de $\mathrm{ST}^{6}$. Los RNPT tienen un mayor riesgo de desarrollar SP por SBHGB con fluctuaciones entre 20 y $39 \%$, triplicándose en los RNPT $\leq 1.500 \mathrm{~g}^{6}$.

La meningitis por SBHGB, puede tener graves consecuencias o discapacidades tales como: deterioro de la visión, pérdida auditiva, retraso del desarrollo psicomotor e intelectual ${ }^{7}$.

La mayoría de las infecciones SBHGB en los recién nacidos $(\mathrm{RN})$, resultan de la transmisión de madre a hijo durante el trabajo de parto o en el parto mismo; sin embargo, esta infección es escasamente patogénica para la mujer y sólo adquiere mayor importancia en el entorno perinatal $^{1,6}$. Se sabe que entre 10 y $30 \%$ de las mujeres embarazadas tienen portación vaginal transitoria, intermitente o persistente durante la gestación; de ellas, $50 \%$ de sus RN se colonizarán por SBHGB al pasar por el canal del parto y de los $\mathrm{RN}$ colonizados sólo $1 \%$ presentará $\mathrm{SP}$ o ST${ }^{8}$. Estudios en mujeres embarazadas en el tercer trimestre en Chile, dan tasas de portación de 14 a 20\% $0^{9,10}$.

En la década de los ochenta, se logró demostrar que la profilaxis antimicrobiana intraparto (PAI), (con ampicilina $u$ hoy en día penicilina), administrada a madres con factores de riesgo de infección neonatal resulta efectiva en prevenir la transmisión de la infección al RN, estableciéndose un período mínimo de cuatro horas previo al parto para la primera dosis de antimicrobiano, dado que con esto se reduce la tasa de colonización del RN a sólo $1,2 \%{ }^{11}$. En el año 1996, en un trabajo conjunto de la American Academy de Pediatría-AAP, el American Congress of Obstetricians and Gynecologists-ACOG y los Centers for Disease Control and Prevention-CDC, se generaron las primeras guías de prevención de la enfermedad por SBHGB estableciéndose dos estrategias igualmente aceptables. La primera es la estrategia de tamizaje universal con cultivo de portación recto-vaginal, a las 35 a 37 semanas de gestación y la segunda, el manejo de las mujeres embarazadas según factores de riesgo clínicos de ser portadoras de SBHGB. Estas guías lograron disminuir en 65 a $80 \%$ la $\mathrm{SP}$ por $\mathrm{SBHGB}^{1,7,11}$.

En el año 2002 el mismo grupo de trabajo, tras una extensa revisión de la evidencia disponible, estableció que la estrategia de tamizaje universal era superior a la de manejo por factores de riesgo, por lo que surgió la recomendación del tamizaje universal en la mujer embarazada entre las 35 y 37 semanas de gestación. Se recomienda la estrategia de PAI según factores de riesgo clínicos, sólo cuando el estado de portación de la mujer 
embarazada fuese desconocido, dado que la mayoría de los RN expuestos a SBHGB al nacer, son hijos de madres colonizadas sin factores de riesgo adicionales ${ }^{11,12}$. Un tercer grupo que debe recibir PAI lo constituyen las mujeres con antecedente de haber tenido antes un RN con infección por SBHGB y/o bacteriuria durante el embarazo actual $^{1}$, estrategia vigente hasta el día de hoy ${ }^{8}$.

A pesar de la amplia implementación del protocolo de PAI con toma de cultivo entre las 35 y 37 semanas, y de la disminución de la incidencia de las infecciones perinatales por SBHGB, éstas (18-21\% actualmente) permanecen como una de las principales causas de admisión a las unidades de cuidados intensivos pediátricos y neonatales y de morbi-mortalidad infecciosa del $\mathrm{RN}^{7}$. De los casos con SP hoy en día, en una experiencia italiana, cerca de 30 a $60 \%$ no recibe PAI porque no califica dentro de las indicaciones establecidas y de los que tienen indicación, apenas 15 a $50 \%$ la recibe correctamente ${ }^{1}$. Las principales causas de la no administración o administración incorrecta de la PAI son: parto precipitado, resistencia a eritromicina y clindamicina en mujeres gestantes alérgicas a $\beta$-lactámicos o la administración iniciada en un tiempo inferior a las $4 \mathrm{~h}$ previas al parto ${ }^{7,13}$. Este fracaso se estima que ocasiona entre 6-13 casos de SP, según un reciente estudio publicado en Hong Kong ${ }^{13}$.

El problema principal de la no administración de la PAI, es el elevado porcentaje de cultivos falsamente negativos en mujeres sin factores de riesgo, lo que ocasiona entre 40 a $60 \%$ de los casos actuales de $\mathrm{SP}^{7,13}$.

En el grupo de mujeres gestantes, que no reciben PAI, ya sea porque el cultivo fue negativo o no presentan factores de riesgo al momento del parto, es necesario incrementar el rendimiento de las técnicas microbiológicas, mediante técnicas de biología molecular al momento del parto, y desarrollar a futuro estrategias de prevención a través de la inmunización ${ }^{7}$.

La portación del SBHGB vaginal es intermitente, por lo tanto, resulta insuficiente la estrategia basada sólo en la detección SBHGB recto-vaginal para definir la indicación de tratamiento antimicrobiano profiláctico durante el trabajo de parto y el parto ${ }^{6,14}$. También se pueden producir falsos negativos del tamizaje por otras causas: muestra insuficiente, transporte inadecuado de las muestras, almacenamiento incorrecto y toma de muestra sólo vaginal y no recto-vaginal (la toma de muestra sólo vaginal no identifica el total de pacientes colonizadas) $)^{2,7}$. A lo anterior se suman los embarazos no controlados y los recién nacidos prematuros que nacen antes de las 35 semanas, que quedan fuera del tamizaje ${ }^{2}$.

Las técnicas de diagnóstico point of care, basadas en la reacción de la polimerasa en cadena (RPC) pueden contribuir a paliar este problema, ya que permiten identificar el SBHGB de forma rápida en el momento del parto. Sin embargo, hasta el momento, aunque ofrecen ventajas como la rapidez, la posibilidad de detectar cepas no hemolíticas o no pigmentadas y tener una sensibilidad y especificidad superiores a $90 \%$, no permiten detectar resistencias a macrólidos y a clindamicima, sumado a los costos involucrados en la implementación de esta técnica. Las recomendaciones aconsejan aplicar estas técnicas en mujeres en las que se desconoce el estado de portación de SBHGB y que no presentan factores de riesgo en el momento del parto. Sin embargo, en las mujeres gestantes con factores de riesgo recomiendan aplicar PAI independientemente del resultado de la RPC. El hecho de conocer de manera rápida intra-parto, que la madre es portadora, puede modificar el manejo del $\mathrm{RN}$ en las primeras 24 h (alargando hasta $48 \mathrm{~h}$ el período de observación y realizando exámenes de evaluación infectológica) y, por lo tanto, contribuir a la prevención de la $\mathrm{SP}^{2,7}$ y también reducir los costos hospitalarios asociados ${ }^{14}$.

Otro problema que surge es la resistencia de SBHGB a clindamicina, la que puede llegar a 30\%. Este elevado porcentaje hace imprescindible la determinación de la susceptibilidad a este antimicrobiano, sobre todo en pacientes alérgicas a $\beta$-lactámicos $\mathrm{y}$, por ende, indica la importancia y el fundamento de que las técnicas de cultivo y biología molecular no son excluyentes, sino que complementarias ${ }^{7}$. Cuando se utilizan ambas técnicas (cultivo y RPC) no se requiere iniciar PAI con el antecedente del cultivo de las 35-37 semanas positivo, si la RPC intra-parto está negativa ${ }^{14}$. La estrategia de incorporar el examen de RPC reduce significativamente el número de tratamientos antimicrobianos inútiles ${ }^{14}$.

La PAI tiene el potencial de afectar el desarrollo temprano de la microbiota intestinal neonatal, en primer lugar modificando la microbiota vaginal y rectal a la que el $\mathrm{RN}$ está expuesto durante el nacimiento y, en segundo lugar debido a la exposición directa del feto a los antimicrobianos, lo que lleva a la desviación de los patrones de colonización normales con alteraciones en la diversidad de la microbiota intestinal del RN, ocasionando a corto plazo alteraciones en el desarrollo de la inmunidad innata y adquirida, esencial en el primer año del $\mathrm{RN}^{15}$, sin considerar los efectos deletéreos a largo plazo (obesidad, asma, etc. $)^{14,15}$.

El desarrollo de una vacuna para SBHGB dirigida a mujeres embarazadas podría potencialmente proporcionar protección a los niños durante el período mayor de vulnerabilidad a la enfermedad invasora (hasta los 3 meses de edad) ${ }^{16}$. Sin embargo, ni siquiera datos sobre estas nuevas estrategias preventivas han sido consistentes en lo que concierne a disminución de la colonización al momento del parto. De hecho, un reciente estudio en fase IB/II donde vacunaron a mujeres embarazadas, demostró mayor porcentaje de colonización al momento del parto que en el tamizaje inicial ${ }^{16}$.

En nuestro país, la estrategia de tamizaje prenatal no ha 
sido incorporada aún en los programas del Ministerio de Salud y no se han publicado estudios de costo-efectividad de estas medidas en el nuestro ni en otros países sudamericanos ${ }^{17}$. En el Manual de Atención Personalizada en el Proceso Reproductivo, MINSAL 2008, que hace alusión a las intervenciones correspondientes al nivel primario y secundario de salud de la mujer embarazada, no se menciona el cultivo de portación recto-vaginal dentro de las actividades a realizar ${ }^{18}$. La Guía Clínica de Prevención del Parto Prematuro, MINSAL 2010, explicita que en mujeres gestantes con menos de 34 semanas con síntomas de parto prematuro y rotura prematura de membranas (RPM) "el cultivo para SBHGB es opcional, ya que toda paciente con RPM de pretérmino debe recibir antibiótico-terapia profiláctica" y en las mujeres con más de 34 semanas, con RPM, no hace alusión al cultivo de portación ${ }^{19}$. La Guía Perinatal, MINSAL 2015, en relación a las infecciones cérvico-vaginales, específicamente la vaginosis bacteriana, menciona a SBHGB como una de las causas y sugiere la toma de cultivo de portación a las mujeres embarazadas a las 35-37 semanas "si la norma local utiliza el cultivo como método de pesquisa"20.

El CCIN de Sociedad Chilena de Infectología recomienda la implementación del tamizaje universal por cultivo recto-vaginal a las 35-37 semanas de gestación a todas las mujeres embarazadas, lo que sumado al manejo por factores de riesgo y/o por antecedentes de infección neonatal por SBHGB en embarazos previos, son las estrategias que contribuyen a disminuir la morbimortalidad por esta infección.

Es necesario establecer políticas de salud que aseguren el financiamiento del tamizaje universal, que permitan realizar este examen en forma obligatoria en todos los centros de salud del país, con oportunidad y calidad, incluyéndolo dentro las prestaciones codificadas por FONASA, de tal manera de asegurar su accesibilidad en forma igualitaria a lo largo del territorio nacional.

\section{Miembros del Comité Consultivo de Infecciones Neonatales:}

Alejandra Sandoval - Hospital Sótero del Río. Fernanda Cofré - Hospital Roberto del Río. Luis Delpiano - Hospital San Borja Arriarán. Giannina Izquierdo - Hospital Exequiel González Cortés. Yenis Labraña - Hospital San Juan de Dios. Alejandra Reyes - Hospital Félix Bulnes Cerda. Mariluz Hernández - Hospital de La Florida. Marta Aravena - Hospital El Carmen.

\section{Referencias bibliográficas}

1.- De Luca C, Buono N, Santillo V, Licameli A, Straface G, Scambia G, et al. Screening and management of maternal colonization with Streptococcus agalactiae: an Italian cohort study. J Matern Fetal Neonatal Med 2016; 29 (6): 911-5.

2.- Reinheimer C, Kempf V A, Wittekindt B E, Allendorf A, Wichelhaus T A, Hogardt M, et al. Group B streptococcus infections in neonates admitted to German NICU: Emphasis on screening and adherence to pre-analytical recommendations. Early Hum Dev 2016; 103 : $37-41$.

3.- Giannoni E, Berger C, Stocker M, Agyeman P, Posfay-Barbe K M, Heininger $U$, et al. Incidence and outcome of Group B Streptococcal sepsis in infants in Switzerland. Pediatr Infect Dis J 2016; 35 (2): 222-4.

4.- Le Doare K, Heath P. An overview of BGS epidemiology. Vaccine 2013; 31 (S4): D7-D12.

5.- Vigilancia de laboratorio enfermedad invasora Streptococcus agalactiae. Boletín Instituto de Salud Pública de Chile 2014; 4 (7): 1-18.

6.- Giménez M, Sanfeliu I, Sierra M, Dopico
E, Juncosa T, Andreu A, et al. Group B streptococcal early-onset neonatal sepsis in the area of Barcelona (2004-2010). Analysis of missed opportunities for prevention. Enferm Infecc Microbiol Clin 2015; 33 (7): 446-50.

7.- Clarke C, O'Connor L, Carré-Skinner H, Piepenburg O, Smith T J. Development and performance evaluation of a recombinase polymerase amplification assay for the rapid detection of group B streptococcus. BMC Microbiol 2016; 16 (1): 221.

8.- Verani J, McGee L, Schrag S. Prevention of perinatal group B Streptococcal disease Revised guidelines from CDC, 2010. Morbid Mortal Wkly Rep 2010; 59: 1-32.

9.- Abarzúa F, Guzmán A M, Belmar C, Becker J, García P, Rioseco A, et al. Prevalencia de colonización por Streptococcus agalactiae en el tercer trimestre del embarazo. Evaluación del cultivo selectivo. Experiencia en 2.192 pacientes. Rev Chil Obstet Ginecol 2002; 67 (2): 89-93.

10.- Abarzúa F, Argomedo C, Meissner A, Díaz T, Garrido P, Fariña S, et al. Prevalencia de portación vaginal-anal de Streptococcus agalactiae en el tercer trimestre de gestación y susceptibilidad a macrólidos y lincosamidas, en mujeres embarazadas de Clínica Alemana Temuco, Chile. Rev Chilena Infectol 2014: 31 (3): 305-8.

11.- Tumbaga P, Philip A. Perinatal group B Streptococcal infections: current status and future directions. Neoreviews 2013; 14: e306e316.

12.- Turrentine M, Greisinger A, Brown K, Wehmanen O, Mouzoon M. Duration on intrapartum antibiotics for Group B Streptococcus on the diagnosis of clinical neonatal sepsis. Inf Dis Obst Gyn 2013, Article ID 525878.

13.- Ma T W, Chan V, So C H, Hui A S, Lee C N, Hui A P, et al. Prevention of early onset group B streptococcal disease by universal antenatal culture-based screening in all public hospitals in Hong Kong. J Matern Fetal Neonatal Med 2017; 21: 1-7.

14.- Poncelet-Jasserand E, Forges F, Varlet M N, Chauleur C, Seffert P, Siani C, et al. Reduction of the use of antimicrobial drugs following the rapid detection of Streptococcus agalactiae in the vagina at delivery by real-time PCR assay. Br J Obstet Gynecol 2013; 120 (9): 1098-108. 
15.- Simioni J, Hutton E K, Gunn E, Holloway A C, Stearns J C, McDonald H, et al. A comparison of intestinal microbiota in a population of low-risk infants exposed and not exposed to intrapartum antibiotics: The Baby \& Microbiota of the Intestine Cohort Study Protocol. BMC Pediatr 2016; 16 (1): 183.

16.- Madhi S A, Cutland C L, Jose L, Koen A,
Govender N, Wittke F, et al. Safety and immunogenicity of an investigational maternal trivalent group B Streptococcus vaccine in healthy women and their infants: a randomised phase $1 \mathrm{~b} / 2$ trial. Lancet Infect Dis 2016; 16 (8): 923-34.

17.- Tapia J, Reinchard C, Saldias I, Abarzúa F, Pérez M, González A, Gederlini A. Sepsis neonatal en la era de profilaxis antimicrobiana prenatal. Rev Chilena Infectol 2007; 24 (2): 111-6.

18.- Manual de atención personalizada en el proceso reproductivo. MINSAL 2008.

19.- Guía Clínica Prevención Parto Prematuro. MINSAL 2010.

20.- Guía Perinatal. MINSAL 2015. 